https://doi.org/10.1007/s10694-021-01142-w

\title{
Correction to: Study on Thermal Response of Adhesively Bonded Honeycomb Sandwich Structure in High Temperature
}

\author{
Rongnan Yuan, Yi Zhang, Yiren Qin and Shouxiang Lu*, State Key \\ Laboratory of Fire Science, University of Science and Technology of China, \\ Hefei 230027, China
}

Published online: 31 May 2021

\section{Correction to:}

Fire Technology

htps://doi.org/10.1007/s1 0694-020-01033-6

The original version of this article unfortunately contained a mistake. The asterisk in the upper right corner of the corresponding author is incorrectly marked for Yiren Qin*. The corresponding author of this article is Shouxiang $\mathrm{Lu}^{*}$. 